\title{
Transversus abdominis plane block as a sole anesthetic technique for evacuation of rectus abdominis muscle hematoma - A case report -
}

Received December 4, 2019

Revised February 6, 2020

Accepted February 6, 2020

\section{Corresponding author}

Jin Sun Kim, M.D.

Department of Anesthesiology and

Pain Medicine, Gangneung Asan

Hospital, University of Ulsan College of Medicine, 38 Bangdong-gil, Sacheonmyeon, Gangneung 25440, Korea

Tel: 82-33-610-3114

Fax: 82-33-641-8180

E-mail: hug77779@gmail.com

\section{Young-Mu Kim, Dong-Min Hyun, Hyun-Soo Kim, and Jin Sun Kim}

Department of Anesthesiology and Pain Medicine, Gangneung Asan Hospital, University of Ulsan College of Medicine, Gangneung, Korea

\begin{abstract}
Background: Transversus abdominis plane (TAP) blocks have been used for analgesia in various abdominal surgeries. However, a TAP block as the sole anesthetic method for surgery has rarely been reported.

Case: A 33-year-old breastfeeding primipara woman was admitted to the hospital due to a rectus abdominis muscle hematoma. Because the patient refused other anesthetic methods, evacuation of the hematoma was performed under an ultrasound-guided bilateral TAP block. A 23-gauge needle was inserted in an in-plane method using a linear ultrasound probe. An injection of $10 \mathrm{ml}$ of $2 \%$ lidocaine was made to the right lateral TAP and the left lateral TAP. After confirming the sensory blockade of the T10 to T12 dermatomes, surgery was performed successfully. The patient's condition stabilized during the surgery. Breastfeeding was performed on the day of surgery.
\end{abstract}

Conclusions: The evacuation of an abdominal wall hematoma in a breastfeeding patient was successfully performed under a bilateral TAP block.

Keywords: Breastfeeding; Hematoma; Nerve block; Ultrasound.
The transversus abdominis plane (TAP) block was first introduced by Rafi [1] in 2001 as an abdominal field block. The TAP block is performed by inserting a needle between the internal oblique muscle and the transversus abdominis muscle and injecting a local anesthetic solution. The TAP block has become multimodal postoperative analgesia for various abdominal surgeries [2]. However, only a few case reports have documented the use of a TAP block alone as a surgical anesthesia, and the anesthetic role of TAP blocks has not yet been clearly defined [3]. According to such case reports, TAP blocks have been adopted as the sole anesthetic technique for high-risk patients who are elderly, have severe systemic diseases, or have contraindications for other methods of anesthesia $[2,4,5]$. This case report differs from the previous case reports in that the TAP block was performed on a nursing primipara woman who refused other anesthesia techniques. In addition, this is the first case report in Korea of surgery using a TAP block as the sole anesthetic technique.

\section{CASE REPORT}

The present case report was approved by the Institutional Review Board of our hospital (no. GNAH 2019-10-003). A 33 -year old female $(82.1 \mathrm{~kg}, 158.1 \mathrm{~cm})$ was admitted to the hospital with abdominal pain and a mass developed after a

This is an Open Access article distributed under the terms of the Creative Commons Attribution Non-Commercial License (http://creativecommons.org/licenses/by-nc/4.0) which permits unrestricted non-commercial use, distribution, and reproduction in any medium, provided the original work is properly cited.

Copyright (C) the Korean Society of Anesthesiologists, 2020 
Cesarean section. Seventeen days earlier, she received a lower segment transverse Cesarean section under spinal anesthesia at a gestational age of $38+2$ weeks with polyhydramnios. An epidural catheter for patient-controlled analgesia (PCA) was placed at L4-5. During the operation, there was no anesthetic or operative complications. After the surgery, a lower rectus abdominis muscle hematoma sized $160.3 \times$ $77.4 \times 91.7 \mathrm{~mm}$ (Fig. 1) was found by abdominal computed tomography, and evacuation of the hematoma was planned.

The patient had no history of severe systemic disease or anticoagulant medication. In the preoperative examination, the complete blood cell count revealed a white blood cell of $11,600 / \mathrm{mm}^{3}$ with an elevated C-reactive protein $(7.48 \mathrm{mg} / \mathrm{dl})$ and fever $\left(38.5^{\circ} \mathrm{C}\right)$. The results of other laboratory and imaging studies, such as electrolytes, blood coagulation tests, chest X-ray, and electrocardiogram (ECG) were all unremarkable.

Prior to the surgery, the patient worried about the accumulation of an anesthetic agent in her breast milk and complained about an unpleasant personal experience with previous spinal anesthesia with epidural catheter insertion. Thus, she refused to receive any neuraxial or general anesthesia. Because of the patient's concern, the surgical team was consulted regarding adequate anesthetic methods. The authors suggested an ultrasound (US)-guided bilateral TAP block and asked the patient for consent and cooperation.

The patient arrived in the operating room and was identified. She was provided with a blood pressure (BP) cuff to monitor her BP and was connected to an ECG machine and pulse oximetry. She was given $2 \mathrm{~L} / \mathrm{min}$ of $\mathrm{O}_{2}$ via nasal prongs throughout the operation. Her initial BP was 114/74 $\mathrm{mmHg}$, pulse rate was $85 / \mathrm{min}$, and $\mathrm{O}_{2}$ saturation was
$100 \%$.

The patient was in the supine position to perform the bilateral TAP block. Before performing the TAP block, the abdominal skin between the iliac crest and the lower costal margin at the mid-axillary line was prepared using betadine and was covered with sterile drapes. The ultrasound probe and cable were covered with a sterile plastic cover. The linear transducer (STERRAD ${ }^{\circledR}$ UST-5413 [5-10 MHz], Hitachi Aloka Medical, Japan) of the ultrasound (Prosound SSD-3500SV, Aloka Co., Japan) was placed on the right mid-axillary line between the subcostal margin and the iliac crest (Fig. 2). With an in-plane approach, the position of the external oblique muscle, internal oblique muscle, and the transverse abdominis muscle were spontaneously checked. A 23-gauge, $60 \mathrm{~mm}$ quincke-type needle (Kovax-needle ${ }^{\circledR}$, Korea Vaccine Co., Korea) was inserted in the fascia layer between the internal oblique muscle and the transverse abdominis muscle to inject $10 \mathrm{ml}$ of $2 \%$ lidocaine (Fig. 3). In the same manner, $10 \mathrm{ml}$ of $2 \%$ lidocaine was injected into the left TAP. The operation proceeded after confirming sensory changes in the T10-T12 dermatomes using the pinprick method 10 min after the TAP block. A $5 \mathrm{~cm}$-sized horizontal incision was made on the scar of the previous Cesarean section, which was at the T12 dermatome. After opening the rectus abdominis fascia, 700 $\mathrm{ml}$ of subfascial hematoma was evacuated. The duration of operation was $30 \mathrm{~min}$ and the patient's condition stabilized during the surgery.

After the operation, the patient was moved to the post-anesthesia care unit and moved to the ward after 30 min without any complaints of surgical pain. The numerical rating scale for pain at the surgical site was assessed for
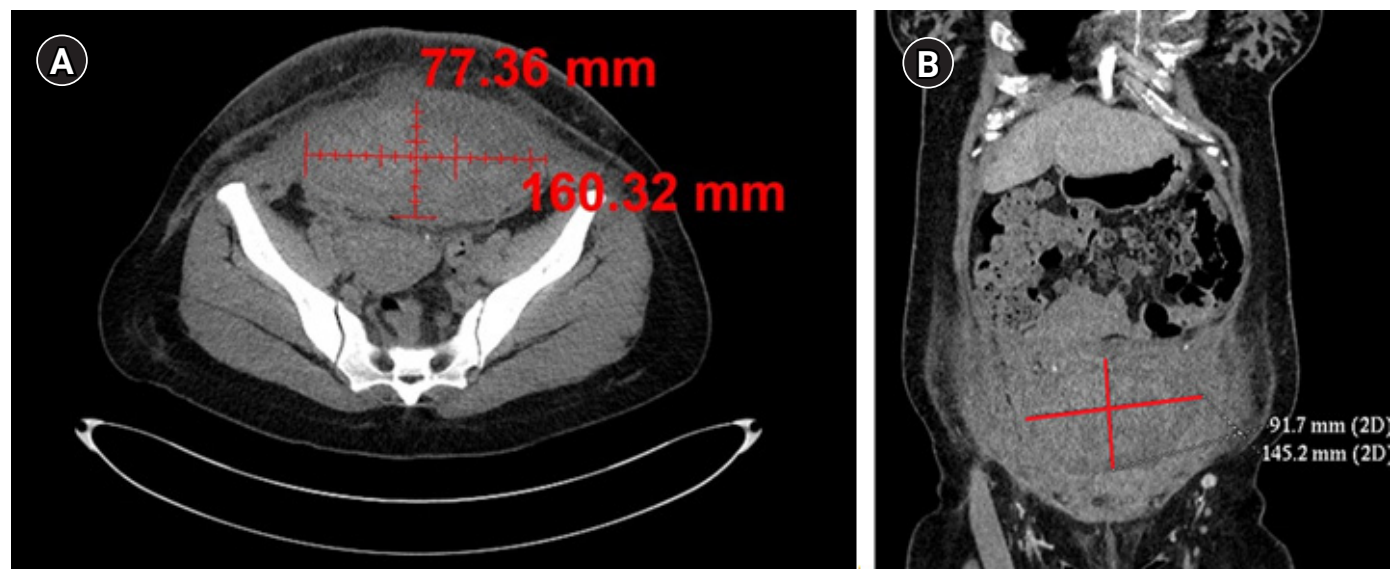

Fig. 1. Lower rectus abdominis muscle hematoma $160.3 \times 77.4 \times 91.7 \mathrm{~mm}$ in size was detected by abdominal computed tomography. It developed after a lower transverse Cesarean section which was performed 17 days ago. (A) Transverse plane image. (B) Coronal plane image. 


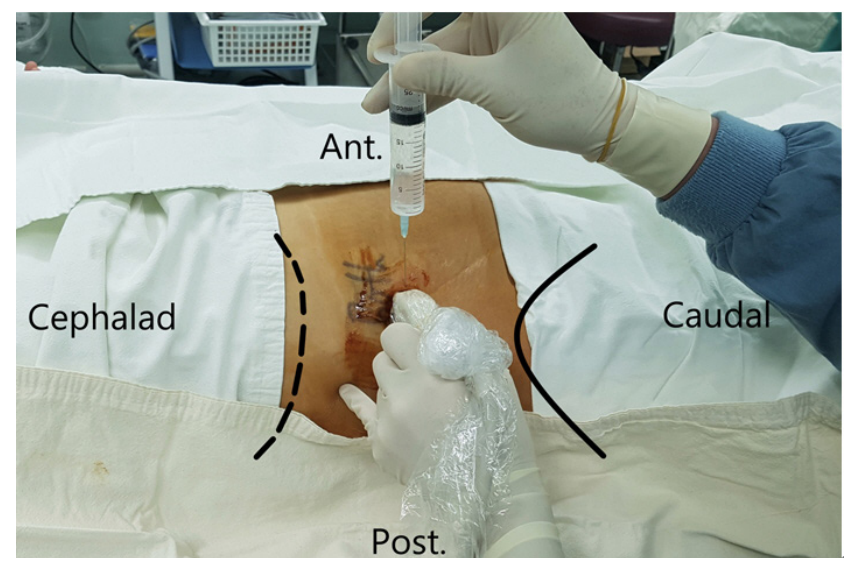

Fig. 2. The patient was in the supine position for the lateral transversus abdominis plane block. The black line indicates the anterolateral part of the iliac crest, right. The black dashed line indicates the lower costal margin. Ant.: anterior, Post.: posterior.

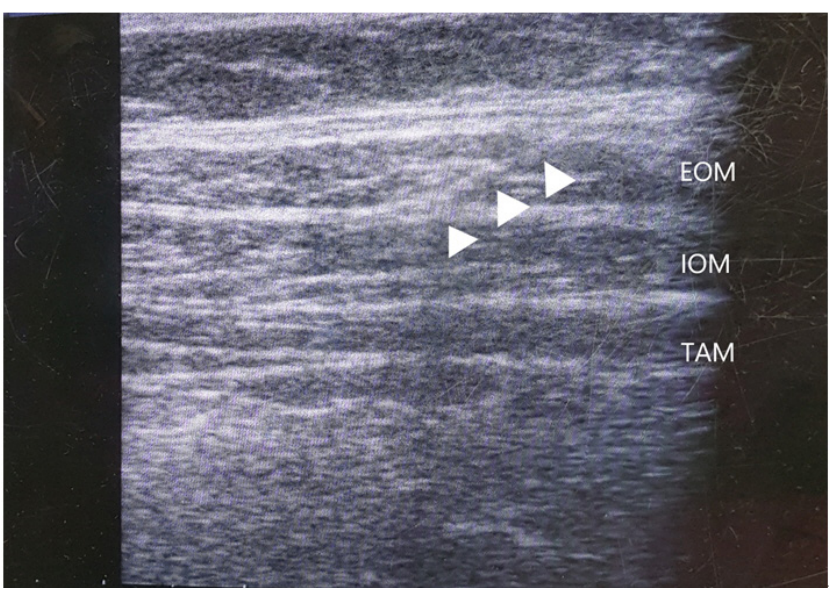

Fig. 3. Ultrasound view of the transversus abdominis plane block after needle insertion. The arrowheads indicate the trajectory of the 23-gauge, $60 \mathrm{~mm}$ quincke-type needle. EOM: external oblique muscle, IOM: internal oblique muscle, TAM: transversus abdominis muscle.

two hours (one hour post-operation, 3 points; two hours post-operation, 5 points) at the ward. The patient showed normal secretion of breast milk and thus, breastfeeding was performed successfully using a breast pump.

\section{DISCUSSION}

Cesarean section is an essential technique for obstetrics, but there are various risks ranging from surgical site problems to life-threatening complications. Wound hematoma is one of the common complications of Cesarean sections. The incidence of wound hematoma in elective Cesarean sections was reported to be $1.3 \%$ and the odds ratio was 5.1 compared to vaginal delivery [6]. The risk factors of rectus muscle hematomas are trauma, surgery, anticoagulation therapy, pregnancy, intense contraction of the rectus muscle, and underlying diseases (hypertension, vasculitis, hematologic disease, and others) [7]. The patient in the present case had no hematologic disease and had never received anticoagulation therapy. However, it is presumed that hematoma could occur due to elevated intra-abdominal pressure by pregnancy and direct/indirect injury of the epigastric vessels during surgery.

Most of the previously published studies introduced TAP blocks only as postoperative analgesic methods. Over the years, the TAP block has proven its analgesic effect in various abdominal surgeries $[2,8]$. TAP blocks can reduce postoperative pain and the need for postoperative opioids for up to $48 \mathrm{~h}$ following abdominal procedures [8,9]. It can be used in combination with PCA or other pain medications, and can also be used as a salvage therapy when other pain medications fail [8]. However, only a few cases of the use of a TAP block as the sole anesthetic technique have been reported. Recently, TAP blocks have been used as the sole anesthetic technique in inguinal hernia repair $[5,10]$, open gastrostomy [3], colectomy [4], ileostomy [4], wedge resection of cecal cancer [4], and abdominal wall abscess incision and drainage [11]. In particular, the last abdominal surgery mentioned above was similar to the present study in the type of surgery and location of the lesion.

Although there are some differences in the literature, the analgesic effect of TAP blocks is generally found in the T6Ll dermatomes of the anterolateral abdominal wall $[1,5,8,12]$. However, a cadaveric study by Tran et al. [13] demonstrated that TAP blocks most effectively blocked the dermatomes of T11-L1 and only 50\% of the T10. In addition, some studies reported that the L1 dermatome was not sufficiently blocked under a posterior TAP block [10]. One of the disadvantages of the TAP block is that it cannot relieve visceral pain mediated by the celiac plexus, which may cause discomfort in surgery stimulating intraperitoneal organs [2]. Although abdominal visceral pain could be controlled by epidural anesthesia at the proper level, the insertion of an epidural catheter was not performed due to the patient's refusal. Because the surgical field of our patient was confined to the lower rectus abdominis muscle without opening the peritoneum, the authors believed that a lateral TAP block could effectively provide sufficient anesthesia for surgery.

Compared to other anesthetic methods, a TAP block has the following advantages. It can be used as a postoperative 
analgesic method $[5,10]$. It reduces hemodynamic fluctuation and the incidence of postoperative nausea and vomiting (PONV) [5] and it contributes to early ambulation after surgery [5]. Moreover, it can be performed in places other than the operating room with or without US [11]. Lastly, even when compared to local anesthesia, TAP blocks provide better and longer pain control with less PONV incidence [3].

However, surgeons should consider the following disadvantages of a TAP block. As mentioned, it is unable to block visceral stimulation and shows an irregular dermatomal range of analgesia [10]. It may cause toxicity due to the use of large amounts of local anesthetics [10] and it takes time to achieve the full analgesic effect after a TAP block, at least 20 to 25 min [2]. Lastly, TAP blocks may provide insufficient analgesia for abdominal midline incisions [14]. Because TAP blocks have many advantages with clear disadvantages, it is necessary to select the appropriate patient and surgical methods carefully before applying a TAP block. Also, it is important to establish adequate communication with surgeons and the patient before surgery.

The patient was a breastfeeding primipara woman with a 17 day-old neonate. She was worried about the accumulation and transmission of anesthetics through the breast milk to her neonate. According to a study that evaluated the delivery of bupivacaine through breast milk after a TAP block [9], neonatal toxicity is extremely unlikely to occur. Local anesthetics, including lidocaine, seem to be safe for breastfeeding women because of their short half-life, poor oral bioavailability (less than $30 \%$ ), and low concentration in breast milk [15]. Most drugs commonly used in general anesthesia are considered to be safe for the breastfeeding of healthy infants when administered in a single dose. But, in some drugs, there have been no published studies on transmission through breast milk (inhaled anesthetics, muscle relaxants, and remifentanil) or only limited data are available. If multiple administration of drugs is needed or a nursing patient has an unhealthy neonate, breastfeeding of the neonate may be adversely affected [15]. In brief, the TAP block is a safe anesthetic method for breastfeeding women, and the patient in the present case was able to proceed to breastfeed normally from the day of the operation.

In conclusion, a breastfeeding primipara woman who rejected other anesthetic methods underwent the successful evacuation of an abdominal wall hematoma under a US-guided bilateral TAP block. A TAP block could be an effective alternative anesthetic method that may cause minimal or no visceral pain in breastfeeding females undergoing abdominal surgery.

\section{CONFLICTS OF INTEREST}

No potential conflict of interest relevant to this article was reported.

\section{AUTHOR CONTRIBUTIONS}

Conceptualization: Young-Mu Kim. Data acquisition: Young-Mu Kim, Dong-Min Hyun, Hyun-Soo Kim. Supervision: Jin Sun Kim. Original draft: Young-Mu Kim. Review \& editing: Young-Mu Kim, Jin Sun Kim.

\section{ORCID}

Young-Mu Kim, https://orcid.org/0000-0002-0286-1010

Dong-Min Hyun, https://orcid.org/0000-0002-3735-7383

Hyun-Soo Kim, https://orcid.org/0000-0001-6817-7214

Jin Sun Kim, https://orcid.org/0000-0002-3753-2997

\section{REFERENCES}

1. Rafi AN. Abdominal field block: a new approach via the lumbar triangle. Anaesthesia 2001; 56: 1024-6.

2. Mishra L, Pani N, Mishra D, Patel N. Bilateral transversus abdominis plane block as a sole anesthetic technique in emergency surgery for perforative peritonitis in a high risk patient. J Anaesthesiol Clin Pharmacol 2013; 29: 540-2.

3. Lee AR, Choe YS. Anesthesia experience for open gastrostomy with ultrasound-guided unilateral subcostal transversus abdominis plane block in a high risk elderly patient: a case report. Anesth Pain Med 2015; 5: e24890.

4. Vuong JT, McQuillan PM, Messaris E, Adhikary SD. Transversus abdominis plane block as the primary anesthetic for laparotomy. J Anaesthesiol Clin Pharmacol 2014; 30: 419-21.

5. Bihani P, Bhatia P, Chhabra S, Gangwar P. Can ultrasound-guided subcostal transverse abdominis plane block be used as sole anesthetic technique? Saudi J Anaesth 2017; 11: 111-3.

6. Liu S, Liston RM, Joseph KS, Heaman M, Sauve R, Kramer MS; Maternal Health Study Group of the Canadian Perinatal Surveillance System. Maternal mortality and severe morbidity associated with low-risk planned cesarean delivery versus planned vaginal delivery at term. CMAJ 2007; 176: 455-60.

7. Hatjipetrou A, Anyfantakis D, Kastanakis M. Rectus sheath hematoma: a review of the literature. Int J Surg 2015; 13: 267-71. 
8. Jankovic Z. Transversus abdominis plane block: the holy grail of anaesthesia for (lower) abdominal surgery. Period Biol 2009; 111: 203-8.

9. Junge J, Inchiosa MA, Xu JL. Exploring the transversus abdominis plane block in cesarean sections and the subsequent toxicity risk to neonates via breast milk. J Anaesthesiol Clin Pharmacol 2019; 35: 153-6.

10. Kerai S, Dabas N, Sehrawat L, Gupta N. Transversus abdominis plane block as sole anaesthetic technique for inguinal hernia repair in two patients having complex medical conditions. Indian J Anaesth 2015; 59: 754-6.

11. Herring AA, Stone MB, Nagdev AD. Ultrasound-guided abdominal wall nerve blocks in the ED. Am J Emerg Med 2012; 30: 759-64.

12. Tsai HC, Yoshida T, Chuang TY, Yang SF, Chang CC, Yao HY, et al. Transversus abdominis plane block: an updated review of anatomy and techniques. Biomed Res Int 2017; 2017: 8284363.

13. Tran TM, Ivanusic JJ, Hebbard P, Barrington MJ. Determination of spread of injectate after ultrasound-guided transversus abdominis plane block: a cadaveric study. Br J Anaesth 2009; 102: 123-7.

14. Støving K, Rothe C, Rosenstock CV, Aasvang EK, Lundstrøm LH, Lange KH. Cutaneous sensory block area, muscle-relaxing effect, and block duration of the transversus abdominis plane block: a randomized, blinded, and placebo- controlled study in healthy volunteers. Reg Anesth Pain Med 2015; 40: 355-62.

15. Oliveira MRE, Santos MG, Aude DA, Lima RME, Módolo NSP, Navarro LH. Should maternal anesthesia delay breastfeeding? A systematic review of the literature. Rev Bras Anestesiol 2019; 69: 184-96. 